\title{
Specialist nurses' perceptions of the barriers and facilitators to inviting adult NHS patients to participate in clinical research studies: a qualitative descriptive study
}

\author{
Caroline French ${ }^{1,2^{*}}$, Charitini Stavropoulou ${ }^{1}$ \\ From 3rd International Clinical Trials Methodology Conference \\ Glasgow, UK. 16-17 November 2015
}

\section{Background}

Specialist nurses often collaborate with researchers to facilitate recruitment of adult NHS patients, yet little is known about their experiences of inviting participation to research studies. It is well documented that clinical staff involved with recruitment often do not invite all potentially eligible patients, leading to underrecruitment and selection bias. Many barriers and facilitators perceived by clinical staff to inviting research participation have been identified, the majority of these relating to physicians or specific randomised controlled trials.

\section{Aims}

This study aims to explore the barriers and facilitators perceived by specialist nurses to inviting adult patients to a variety of research studies in different NHS settings.

\section{Methods}

The study employs a cross-sectional qualitative descriptive design using purposive and convenience sampling, semi-structured individual interviews and Framework thematic analysis. This study forms an MRes student dissertation and will be completed in September 2015. To date 12 specialist nurses have been recruited, representing a variety of clinical specialties and employing NHS Trusts, with experience of inviting patients to a broad variety of research studies. Data collection is ongoing and 6 interviews have been conducted thus far.

\section{Results}

Emerging preliminary themes include the perceived facilitator of rapport and clinical credibility within the nurse / patient relationship, and the perceived barrier of insufficient understanding of individual research studies by the nurse.

\section{Conclusions and implications}

Increased understanding of both barriers and facilitators may inform development of evidence-based interventions to optimise research recruitment involving specialist nurses and other healthcare staff.

\section{Authors' details}

${ }^{1}$ City University, London, UK. ${ }^{2}$ Solent NHS Trust, Southampton, UK.

Published: 16 November 2015

doi:10.1186/1745-6215-16-S2-P94

Cite this article as: French and Stavropoulou: Specialist nurses' perceptions of the barriers and facilitators to inviting adult NHS patients to participate in clinical research studies: a qualitative descriptive study. Trials 2015 16(Suppl 2):P94.

${ }^{1}$ City University, London, UK

Full list of author information is available at the end of the article 